Adv Exp Med Biol. 2012 ; 723: 513-518. doi:10.1007/978-1-4614-0631-0_64.

\title{
Biology of Retinoschisin
}

Camasamudram Vijayasarathy,

Section for Translation Research in Retinal and Macular Degeneration, National Institute on Deafness and Other Communication Disorders, National Institutes of Health, Bethesda, MD 20892, USA

Lucia Ziccardi, and

Neurophthalmology Unit, Fondazione “G.B. Bietti” IRCCS, Via Livenza, 300198 Rome, Italy

Paul A. Sieving

National Eye Institute, National Institutes of Health, Bethesda, MD 20892, USA

Paul A. Sieving: paulsieving@nei.nih.gov

\section{Keywords}

X-linked retinoschisis; Retinoschisin; Discoidin domain; Mutations; Retina; Photoreceptors; Bipolar cells; Synapse; Gene therapy

\subsection{Introduction}

The vertebrate retina is a light sensitive neural tissue lining the inner surface of the eye. The basic functional unit of the retina is comprised of photoreceptors, bipolar, horizontal, amacrine, and ganglion cells, which, in each point in the visual field, operate to capture light, process and integrate visual information before delivering it to the brain. The sequential positioning of the cellular components through the retinal depth, and the parallel arrangements of many such basic units across the entire retina contribute to the creation of a mosaic pattern. Many studies reveal an important role of adhesion molecules and their binding partners in controlling retinal patterning (Galli-Resta et al. 2008). Retinoschisin (RS1) is one of the proteins implicated in maintenance of retinal architecture. In young males, loss of RS1 function due to mutations in the X-linked retinoschisis gene (RS1; MIM: 312700) leads to splitting within the retinal layers, which impairs visual-signal processing and leads to progressive vision loss in the condition known as X-linked juvenile retinoschisis (XLRS) (Sauer et al. 1997).

\subsection{RS1 Gene and Protein}

The human RS1 gene on the X-chromosome spans $32.43 \mathrm{~kb}$ of DNA, consisting of six exons that encode a 12-kb mRNA (NM_000330.3*) which is translated into a 224 amino acid retina-specific secretory protein, RS1 (NP_000321.1). RS1 is prominently expressed by the rod and cone inner segments, including the foveal and macular cone photoreceptors, and bipolar cells (Molday et al. 2001; Reid et al. 2003). RS1 expression occurs early in postnatal development of the mouse retina and by $\mathrm{P} 7$ is apparent in the newly formed outer plexiform layer (OPL) (Takada et al. 2004). Pinealocytes, which are evolutionarily related to the photoreceptors, also express RS1, but the role in the pineal gland is not known (Takada et al.

(C) Springer Science+Business Media, LLC 2012

Correspondence to: Paul A. Sieving, paulsieving@nei.nih.gov. 
2006). The 24-kDa RS1 protein encodes two functional sites of conserved sequence motifs, the $\mathrm{N}$-terminus signal sequence (aa 1-21/23) that directs protein translocation to the exterior of the cell, and a long and highly conserved sequence motif termed the discoidin domain (exons 4-6; aa 64-219) (Wu et al. 2005; Vijayasarathy et al. 2006). The discoidin domain apparently contributes to the adhesive function of RS1 to preserve the retinal cell architecture and to establish proper synaptic connectivity (Takada et al. 2008). RS1 is a peripheral membrane protein bound by ionic forces to the outer leaflet of the photo-receptor inner segment plasma membrane (Vijayasarathy et al. 2007). The functional conformation of RS1 is an octamer with eight subunits joined together by Cys(59)-Cys(223) intermolecular disulfide bonds (Wu et al. 2005). Subunits within the octamer are further organized into dimers mediated by Cys(40)-Cys(40) bonds.

\subsection{RS1 Interactions}

RS1 binds specifically to the anionic phospholipids for example, phosphatidylserine (PS) in the presence of $\mathrm{Ca}^{2+}$ (Vijayasarathy et al. 2007). Several other possible RS1 interacting ligands were identified: $\mathrm{Na}^{+} / \mathrm{K}^{+}$ATPase and the sterile alpha and TIR motif-containing protein, SARM1 (Molday et al. 2007), alphaB crystallin and beta2 laminin (SteinerChampliaud et al. 2006), and L-type voltage-gated calcium channel (LVGCC) (Shi et al. 2009). However, the structural basis for these interactions has not been defined. Imaging with atomic force microscopy provided biophysical evidence that RS1 causes major reorganization of PS-containing supported bilayers in the presence of calcium cations (Kotova et al. 2010). Membrane proteins often preferentially reside and function optimally within lipid domains enriched in certain types of lipids, and it is likely that a lipid-binding protein, such as RS1, may act to stabilize membrane microdomains into which membrane proteins assemble, such as $\mathrm{Na}^{+} / \mathrm{K}^{+}$ATPase, SARM, and LVGCC. RS1 bound by phospholipids on the membrane surface can form a multimolecular stabilizing scaffold that participates in cell-matrix, cell-cell interactions, and cytoskeleton organization.

\subsection{X-Linked Retinoschisis Mutations}

XLRS patients harbor a wide spectrum of $R S 1$ mutations, with the majority occurring in the discoidin domain. Mutations are known to occur across the entire gene, including both the introns and the exons, and include missense, nonsense, frame-shift (deletions/insertions), and splice-site mutations (The Retinoschisis Consortium: http://www.dmd.nl/rs/ consortium.html). The molecular consequences have been shown to affect RS1 biosynthesis in multiple ways, including the processing of pre-RNA transcript, translation initiation, ability to mature in the secretory pathway, and the ability to achieve a biologically active octamer conformation due to mis-folding (Wu and Molday 2003; Wang et al. 2006; Vijayasarathy et al. 2009, 2010). Two major biochemical signatures emerge from these point mutations: an RS1-null phenotype results from an absolute lack of RS1 protein, whereas others give a signature of functionally incompetent misfolded mutant RS1 molecules. While some misfolded proteins (Leu12His and Leu13Pro) are disposed by the large ATPdependent proteolytic machine involving the $26 \mathrm{~S}$ proteasome (endoplasmic reticulumassociated degradation), other misfolded mutants (Glu72Lys, Arg102Trp, Arg213Trp, Asn179Asp, and Pro192S) are stabilized in the cell and form high molecular mass aggregates (Vijayasarathy et al. 2010).

\subsection{Clinical Pathology}

XLRS is transmitted in an X-linked recessive pattern that causes male-only disease, while the female carriers have no symptoms. In rare cases, skewed X-chromosome inactivation leads to XLRS phenotype heterozygous females (Rodriguez et al. 2005). Historically, XLRS lesions were thought to be related to a defect in Müller cells, which span the thickness of the 
retina (Mooy et al. 2002). The principle clinical pathology in XLRS includes vitreo-retinal dystrophy characterized by splitting of inner retinal layers and alterations of the vitreous body, peripheral retinal schisis with vitreous veils, inner retinal holes and tractions in half of the affected males, and a selective b-wave reduction on electroretinogram (ERG) recordings (George et al. 1996; Tantri et al. 2004; Sikkink et al. 2007; Lesch et al. 2008; Vijayasarathy et al. 2009). Abnormalities in the ERG a-wave have been observed at least in some patients (Bradshaw et al. 1999) but in many ERG a-wave remains normal (Khan et al. 2001). The stellate spoke-like maculopathy with microcysts seen at young ages may progress to macular atrophy during the third to fifth decades of life (Vijayasarathy et al. 2009). Patients experience an early onset central vision reduction. Schisis cavities may lead to ruptured vessels and vitreous bleeds, or retinal detachments may occur. XLRS patients display a particularly high clinical variability with a remarkably broad spectrum of phenotypes (Hiriyanna et al. 2001).A strict correspondence between genetic mutations and clinical severity has been sought but not found, although males harboring RS1 missense mutations seem to present a less severe phenotype than those carrying null mutations (Bradshaw et al. 1999; Pimenides et al. 2005; Vijayasarathy et al. 2009, 2010; Sergeev et al. 2010). Genetic factors such as tyrosinase gene (Tyr) that modulate severity and disease penetrance have been mapped in mouse models of XLRS (Johnson et al. 2010) but have not been identified for human XLRS.

\subsection{Molecular Pathology}

Knockout mouse models have been helpful in deducing the molecular mechanisms of the retinal degeneration and in evaluating the strategies for gene therapy. The $R S 1$ knockout mice share several important clinical features with human XLRS disease (Weber et al. 2002; Zeng et al. 2004; Jablonski et al. 2005; Kjellstrom et al. 2007). Consistent with a role for RS1 in maintaining photoreceptor stability, electron microscopy showed profound pathological alterations in the morphology of rod inner segments in the absence of RS1 protein (Johnson et al. 2006; Takada et al. 2008). The pre- and postsynaptic processes, along with synaptic terminal proteins (PSD 95 and mGluR6), were displaced from the OPL into the outer nuclear layer, and rod nuclei can be shifted into the inner segment region. Reduced areal density of synaptic vesicles at the photoreceptor presynapse was also found. Bipolar and photoreceptor cells both express RS1. Despite the deficiency of RS1 in Rs1-KO bipolar cells, the inner plexiform layer appeared morphologically intact, suggesting that bipolar axons are targeted correctly in the proximal retina. These findings imply that (a) RS1 acts locally to maintain the photoreceptor inner segment stability and architecture; (b) the loss of integrity of OPL, inner nuclear layer, and photoreceptor cells is a major pathologic feature of the retina of the Rs1-KO mice; (c) RS1 appears to be needed for preservation of synaptic structures but not for synaptogenesis; (d) early effects on synaptic structure contribute to the functional deficit reflected in the b-wave reduction; and (e) axonal degeneration or axonal retraction stemming from a defect in photoreceptor homeostasis may contribute to synaptic loss in XLRS disease.

\subsection{Prospects for Gene Therapy}

Lack of a dominant-negative effect of XLRS mutations in carrier females provides evidence that gene transfer may be successful for XLRS patients. The RS1 gene was introduced into the mouse models of XLRS both by a subretinal injection approach (AAV5-RS1 vector using opsin promoter) and through intravitreal delivery of AAV8- $R S 1$ vector using a $R S 1$ promoter (Janssen et al. 2008; Park et al. 2009) at different stages of disease: early at P15, P30, P60 or late at 7 months. The vitreal route for $R S 1$ gene administration would be safer than subretinal injection which requires retinal surgical manipulation and vitrectomy. Overall, the gene therapy treatments in XLRS mice lead to improvement in both retinal ERG 
function and morphology, and the rate and extent of degeneration were less pronounced in the treated eye than in the untreated eye of the same animal (Park et al. 2009). Gene transfer at advanced stages of the disease ( 7 months of age) showed poor response (Janssen et al. 2008). Treatment with AAV8-h $R S \mathrm{p} 4-R S 1$ vector by vitreal administration resulted in robust tissue-specific RS1 expression in photoreceptor inner segments lying deep in the retina (Park et al. 2009). Most importantly, cellular expression of RS1 was correctly targeted with this vector and was not seen in ocular tissues that do not normally express RS1 (optic nerve, retinal fiber layer, peripheral cornea, or ciliary body). The results hold promise for future clinical trials in human patients and as a new therapeutic strategy for XLRS treatment.

\section{References}

Bradshaw K, George N, Moore A, et al. Mutations of the XLRS1 gene cause abnormalities of photoreceptor as well as inner retinal responses of the ERG. Doc Ophthalmol. 1999; 98:153-173. [PubMed: 10947001]

Galli-Resta L, Leone P, Bottari D, et al. The genesis of retinal architecture: an emerging role for mechanical interactions? Prog Retin Eye Res. 2008; 27:260-283. [PubMed: 18374618]

George ND, Yates JR, Moore AT. Clinical features in affected males with X-linked retino-schisis. Arch Ophthalmol. 1996; 114:274-280. [PubMed: 8600886]

Hiriyanna, KT.; Singh-Parikshak, R.; Bingham, EL., et al. Searching for genotype-phenotype correlations in X-linked juvenile retinoschisis. In: Anderson, RELVM.; Hollyfield, JG., editors. New Insights Into Retinal Degenerative Diseases. New York: Plenum Publishers; 2001. p. 45-53.

Jablonski MM, Dalke C, Wang X, et al. An ENU-induced mutation in Rs1h causes disruption of retinal structure and function. Mol Vis. 2005; 11:569-581. [PubMed: 16088326]

Janssen A, Min SH, Molday LL, et al. Effect of late-stage therapy on disease progression in AAVmediated rescue of photoreceptor cells in the retinoschisin-deficient mouse. Mol Ther. 2008; 16:1010-1017. [PubMed: 18388913]

Johnson BA, Ikeda S, Pinto LH, Ikeda A. Reduced synaptic vesicle density and aberrant synaptic localization caused by a splice site mutation in the Rs1h gene. Vis Neurosci. 2006; 23:887-898. [PubMed: 17266781]

Johnson BA, Cole BS, Geisert EE, et al. Tyrosinase is the modifier of retinoschisis in mice. Genetics. 2010; 186:1337-1344. [PubMed: 20876567]

Khan NW, Jamison JA, Kemp JA, et al. Analysis of photoreceptor function and inner retinal activity in juvenile X-linked retinoschisis. Vision Res. 2001; 41:3931-3942. [PubMed: 11738458]

Kjellstrom S, Bush RA, Zeng Y, et al. Retinoschisin gene therapy and natural history in the Rs1h-KO mouse: long-term rescue from retinal degeneration. Invest Ophthalmol Vis Sci. 2007; 48:38373845. [PubMed: 17652759]

Kotova S, Vijayasarathy C, Dimitriadis EK, et al. Retinoschisin (RS1) interacts with negatively charged lipid bilayers in the presence of $\mathrm{Ca} 2+$ : an atomic force microscopy study. Biochemistry. 2010; 49:7023-7032. [PubMed: 20677810]

Lesch B, Szabo V, Kanya M, et al. Clinical and genetic findings in Hungarian patients with X-linked juvenile retinoschisis. Mol Vis. 2008; 14:2321-2332. [PubMed: 19093009]

Molday LL, Wu WW, Molday RS. Retinoschisin (RS1), the protein encoded by the X-linked retinoschisis gene, is anchored to the surface of retinal photoreceptor and bipolar cells through its interactions with a Na/K ATPase-SARM1 complex. J Biol Chem. 2007; 282:32792-32801. [PubMed: 17804407]

Molday LL, Hicks D, Sauer CG, et al. Expression of X-linked retinoschisis protein RS1 in photoreceptor and bipolar cells. Invest Ophthalmol Vis Sci. 2001; 42:816-825. [PubMed: 11222545]

Mooy CM, Van Den Born LI, Baarsma S, et al. Hereditary X-linked juvenile retinoschisis: a review of the role of Muller cells. Arch Ophthalmol. 2002; 120:979-984. [PubMed: 12096974]

Park TK, Wu Z, Kjellstrom S, et al. Intravitreal delivery of AAV8 retinoschisin results in cell typespecific gene expression and retinal rescue in the Rs1-KO mouse. Gene Ther. 2009; 16:916-926. [PubMed: 19458650] 
Pimenides D, George ND, Yates JR, et al. X-linked retinoschisis: clinical phenotype and RS1 genotype in 86 UK patients. J Med Genet. 2005; 42:e35. [PubMed: 15937075]

Reid SN, Yamashita C, Farber DB. Retinoschisin, a photoreceptor-secreted protein, and its interaction with bipolar and muller cells. J Neurosci. 2003; 23:6030-6040. [PubMed: 12853421]

Rodriguez FJ, Rodriguez A, Mendoza-Londono R, et al. X-linked retinoschisis in three females from the same family: a phenotype-genotype correlation. Retina. 2005; 25:69-74. [PubMed: 15655444]

Sauer CG, Gehrig A, Warneke-Wittstock R, et al. Positional cloning of the gene associated with Xlinked juvenile retinoschisis. Nat Genet. 1997; 17:164-170. [PubMed: 9326935]

Sergeev YV, Caruso RC, Meltzer MR, et al. Molecular modeling of retinoschisin with functional analysis of pathogenic mutations from human X-linked retinoschisis. Hum Mol Genet. 2010; 27:27.

Shi L, Jian K, Ko ML, et al. Retinoschisin, a new binding partner for L-type voltage-gated calcium channels in the retina. J Biol Chem. 2009; 284:3966-3975. [PubMed: 19074145]

Sikkink SK, Biswas S, Parry NR, et al. X-linked retinoschisis: an update. J Med Genet. 2007; 44:225232. [PubMed: 17172462]

Steiner-Champliaud MF, Sahel J, Hicks D. Retinoschisin forms a multi-molecular complex with extracellular matrix and cytoplasmic proteins: interactions with beta2 laminin and alphaBcrystallin. Mol Vis. 2006; 12:892-901. [PubMed: 16917482]

Takada Y, Vijayasarathy C, Zeng Y, et al. Synaptic pathology in retinoschisis knockout (Rs1-/y) mouse retina and modification by rAAV-Rs1 gene delivery. Invest Ophthalmol Vis Sci. 2008; 49:3677-3686. [PubMed: 18660429]

Takada Y, Fariss RN, Muller M, Bush RA, Rushing EJ, Sieving PA. Retinoschisin expression and localization in rodent and human pineal and consequences of mouse RS1 gene knockout. Mol Vis. 2006; 12:1108-1116. [PubMed: 17093404]

Takada Y, Fariss RN, Tanikawa A, et al. A retinal neuronal developmental wave of retino-schisin expression begins in ganglion cells during layer formation. Invest Ophthalmol Vis Sci. 2004; 45:3302-3312. [PubMed: 15326155]

Tantri A, Vrabec TR, Cu-Unjieng A, et al. X-linked retinoschisis: a clinical and molecular genetic review. Surv Ophthalmol. 2004; 49:214-230. [PubMed: 14998693]

Vijayasarathy C, Takada Y, Zeng Y, et al. Retinoschisin is a peripheral membrane protein with affinity for anionic phospholipids and affected by divalent cations. Invest Ophthalmol Vis Sci. 2007; 48:991-1000. [PubMed: 17325137]

Vijayasarathy C, Gawinowicz MA, Zeng Y, et al. Identification and characterization of two mature isoforms of retinoschisin in murine retina. Biochem Biophys Res Commun. 2006; 349:99-105. [PubMed: 16930543]

Vijayasarathy C, Ziccardi L, Zeng Y, et al. Null retinoschisin-protein expression from an RS1 c354del1-ins 18 mutation causing progressive and severe XLRS in a cross-sectional family study. Invest Ophthalmol Vis Sci. 2009; 50:5375-5383. [PubMed: 19474399]

Vijayasarathy C, Sui R, Zeng Y, et al. Molecular mechanisms leading to null-protein product from retinoschisin (RS1) signal-sequence mutants in X-linked retinoschisis (XLRS) disease. Hum Mutat. 2010; 31:1251-1260. [PubMed: 20809529]

Wang T, Zhou A, Waters CT, O'Connor E, et al. Molecular pathology of X linked retinoschisis: mutations interfere with retinoschisin secretion and oligomerisation. Br J Ophthalmol. 2006; 90:81-86. [PubMed: 16361673]

Weber BH, Schrewe H, Molday LL, et al. Inactivation of the murine X-linked juvenile retinoschisis gene, Rs1h, suggests a role of retinoschisin in retinal cell layer organization and synaptic structure. Proc Natl Acad Sci USA. 2002; 99:6222-6227. [PubMed: 11983912]

Wu WW, Molday RS. Defective discoidin domain structure, subunit assembly, and endoplasmic reticulum processing of retinoschisin are primary mechanisms responsible for X-linked retinoschisis. J Biol Chem. 2003; 278:28139-28146. [PubMed: 12746437]

Wu WW, Wong JP, Kast J, Molday RS. RS1, a discoidin domain-containing retinal cell adhesion protein associated with $\mathrm{X}$-linked retinoschisis, exists as a novel disulfide-linked octamer. J Biol Chem. 2005; 280:10721-10730. [PubMed: 15644328] 
Zeng Y, Takada Y, Kjellstrom S, et al. RS-1 Gene Delivery to an Adult Rs1h Knockout Mouse Model Restores ERG b-Wave with Reversal of the Electronegative Waveform of X-Linked Retinoschisis. Invest Ophthalmol Vis Sci. 2004; 45:3279-3285. [PubMed: 15326152] 